\title{
COMPORTAMIENTO DE ENFERMEDADES EPIDÉMICAS A TRAVÉS DEL MODELO MATEMÁTICO SIR: UNA REVISIÓN DE LA LITERATURA
}

\author{
Oswaldo Daniel Casazola Cruz \\ odcasazolac@unac.edu.pe \\ https://orcid.org/0000-0003-2521-530X \\ Alex Manuel Apaza Mendoza \\ alexapazamendoza@gmail.com \\ Mixcel Fiorella Julca Pillman \\ fiorejulcapillman@gmail.com \\ Carlos Roberto Delgado Lopez \\ carlosrobertodelgadolopez@gmail.com \\ Universidad Nacional del Callao, Perú
}

\section{Resumen}

El artículo describe las tendencias del comportamiento de enfermedades epidémicas basándose en el modelo epidemiológico SIR. El objetivo de este trabajo fue describir el comportamiento de enfermedades epidémicas basándose en el modelo epidemiológico SIR a partir de la revisión de la literatura bajo la metodología de la revisión sistemática con base en la estrategia Prisma. Los resultados mostraron el uso del modelo epidemiológico SIR para describir el comportamiento de varias enfermedades de naturaleza epidémica, ahondando en mayor frecuencia la enfermedad producida por el COVID-19 en los últimos años. Se concluye la importancia del uso del modelo SIR para la toma de decisiones como medidas de contención en la propagación de la enfermedad, así como el uso de un indicador importante como el índice de reproducción básica para determinar el comportamiento de la enfermedad.

PALABRAS CLAVE: modelo SIR / COVID-19 / enfermedades / epidémicas / coronavirus / modelos epidemiológicos 


\section{THE BEHAVIOUR OF EPIDEMIC DISEASES THROUGH THE SIR MATHEMATICAL MODEL: A REVIEW OF THE LITERATURE}

\section{Abstract}

The article describes the trends in the behavior of epidemic diseases based on the SIR epidemiological model. The objective of this work was to describe the behavior of epidemic diseases based on the SIR epidemiological model from the review of the literature under the methodology of the systematic review based on the PRISMA strategy. The results showed the use of the SIR epidemiological model to describe the behavior of various diseases of an epidemic nature, deepening the disease produced by COVID-19 in recent years in greater frequency. Concluding with the importance of using the SIR model for decision-making as containment measures in the spread of the disease, and the use of an important indicator such as the basic reproduction index to determine the behavior of the disease.

KEYWORDS: SIR model / COVID-19 / diseases epidemic / coronavirus / epidemiological models 


\section{INTRODUCCIÓN}

A lo largo de la historia de la humanidad han ido apareciendo diferentes enfermedades, en especial enfermedades virales, que se propagaron velozmente y como consecuencia traían consigo muertes y grandes impactos en diversos sectores en el mundo. Sus inicios datan desde la civilización egipcia, en la que se desarrolló la peste bubónica; así también como la aparición de las plagas de Egipto, donde se atribuía a la aparición de epidemias como consecuencia de la cólera divina. Además, en la civilización griega la aparición de la peste en Atenas y la tifoidea en Siracusa (Pesco, 2017).

Autores como Grillo Ardila, Gutiérrez y otros, en el año 2020, así como Pedersen en su tesis desarrollada en el 2015 , señalan que a lo largo de la historia han ido apareciendo modelos matemáticos desarrollados para entender y modelar el comportamiento de enfermedades endémicas que han ido surgiendo en diversas partes del mundo (Grillo et al., 2020; Gutiérrez y Varona, 2020).

En la actualidad estamos inmersos en una nueva pandemia, la Organización Mundial de la Salud (OMS) reportó la aparición de un nuevo virus denominado SARS-CoV-2 el 21 de enero del 2020 (WHO, 2020). Desde esa fecha la infección se ha expandido en todo el mundo llegando a convertirse en una pandemia (WHO, 2020).

Los autores Morens, Folkers y Fauci, citado en Speranza y Masci (2020), exponen a la definición de pandemia como una epidemia distribuida sobre un área suficientemente grande, con un movimiento geográfico de la enfermedad, con elevadas tasas de contagio de crecimiento exponencial y ante una mínima población que genera inmunidad.

En los últimos veinte años, las infecciones causadas por coronavirus tipo $\beta$ como el SARS-CoV-1 y el MERS-CoV han alcanzado tasas de mortalidad del $10 \%$ y $37 \%$ respectivamente. En el caso del coronavirus, enfermedad producida por el virus SARS-CoV-2, se detectaron más de seis millones de infectados y cerca de trescientos mil fallecidos alrededor del mundo entre finales del 2019 y mayo del 2020 (Wilches y Castillo, 2020).

Para algunos autores como Catano-Lopez, Manrique-Abril, Wilches Viscal y Castillo Pedraza, la enfermedad producida por el nuevo coronavirus (COVID-19) es una infección que afecta a las vías respiratorias y que se transmite de persona a persona. Fue identificada por primera vez durante un brote ocurrido en la ciudad de Wuhan en China en el mes de diciembre del año 2019. El causante de la enfermedad es el virus SARS-CoV-2, un coronavirus tipo $\beta$ que consta de una cadena de ARN protegida por una envoltura esférica de 60-140 nm de diámetro. A pesar de que gran parte de las infecciones causadas por coronavirus son leves, las relacionadas con los coronavirus tipo $\beta$ como el SARS-CoV- 1 (síndrome respiratorio agudo severo) y el MERS-CoV (síndrome respiratorio del Medio Oriente) alcanzaron una tasa de mortalidad del $10 \%$ y $37 \%$, respectivamente, en las dos 
últimas décadas (Wilches y Castillo, 2020; Catano-Lopez y Rojas-Diaz, 2020; ManriqueAbril et al., 2020).

Sobre la base a lo descrito, la modelación de epidemias es una herramienta importante para el estudio de enfermedades emergentes ya que permite conocer su comportamiento y evolución, todo ello para poder determinar medidas de control óptimas para la supresión de la enfermedad (Pliego, 2011).

Los científicos Kermack y Mc Kendrick en el año 1927, citados en Pesco (2017), publican un artículo en el cual presentan un modelo matemático basado en ecuaciones diferenciales que simula la transmisión de una enfermedad infecciosa. Como lo explican autores como Salvador Galindo, Gonzales-Castillo, Sepúlveda y otros, según el estado epidemiológico de los individuos, la población está dividida en compartimientos, en los cuales hay una interacción entre la infección, recuperación y fallecidos (GonzalesCastillo, 2020; Galindo, 2020; Sepúlveda, 2015).

Como señalan autores como Cabo Bizet, Peso, Pino-Romero y Vergara-Moreno acerca del modelo SIR, este modelo basado en compartimientos permite simular la transmisión de una enfermedad infecciosa presente en una población realizando hipótesis simples y realistas en base a su transmisión. Además, señalan que este modelo permite obtener información de algunas de las características generales de las diferentes enfermedades infecciosas, como lo es su comportamiento durante la epidemia, los picos epidémicos periódicos, etc. El modelo SIR es uno de los modelos más simples y estudiados a lo largo del tiempo, debido a que se pueden ir agregando nuevos procesos y características al modelo, puede ser difícil implementarlos de manera matemática. No obstante, las variables modeladas en este modelo tienen un comportamiento similar a las de otros modelos más complejos, por lo que su uso es importante para la comprensión de la dinámica de la enfermedad, así como el poder interpretar y contrastar los resultados (Pesco, 2017; Pino et al., 2020; Vergara et al., 2020; N. Cabo y A. Cabo, 2020).

Algunos autores indican que "un modelo matemático es una descripción, en lenguaje matemático, de un objeto que existe en un universo no-matemático" (Yanqui, 2018).

La modelación matemática de enfermedades cuenta con dos objetivos principales, que son la predicción y la comprensión del fenómeno. Estos dos objetivos puede que entren en conflicto ya que, en la predicción se buscará incluir variables en el modelo para tener mayor precisión; por otro lado, para la comprensión del fenómeno se busca tener solo las variables más importantes que influyen en el modelo (Yanqui, 2018).

Con lo señalado por Yanqui Díaz, Pliego menciona que la modelación de epidemias es una herramienta importante para el estudio de enfermedades emergentes ya que permite conocer su comportamiento y evolución, todo ello para poder determinar medidas de control óptimas para la supresión de la enfermedad (Pliego, 2011). 
Con la llegada del SARS-CoV-2, los países de todo el mundo han sido afectados y duramente golpeados debido a la escasez de información y el desconocimiento del comportamiento de la enfermedad, trayendo consigo lamentables pérdidas humanas como impactos en sectores sociales como económicos. El adecuado estudio del modelo SIR aplicando técnicas permite tener un mayor conocimiento de la evolución del SARS-CoV-2 y así se pueda tener un soporte en la toma de decisiones por parte de las autoridades.

A raíz de ello, muchas investigaciones se han venido desarrollando acerca del modelamiento del COVID-19 haciendo uso del modelo SIR. En el Perú, artículos científicos realizados en la Universidad Nacional de Trujillo que tienen como objetivo modelar el comportamiento de la epidemia, han llegado a la conclusión de que el modelo SIR permite analizar la evolución del COVID-19 y que a partir de la data brindada por las autoridades se tienen resultados en el modelo con un margen de error menor y que ayuda a la toma de decisiones (Pino et al., 2020; Vergara et al., 2020).

Otras investigaciones realizadas en países de América Latina como Cuba, Argentina, República Dominicana y Colombia implementan el modelo SIR para describir el comportamiento del COVID-19, y concluyen que el modelo describe con una exactitud aceptable el comportamiento, permitiendo que las autoridades tengan un soporte en la toma de decisiones con medidas de salud pública (Abelló et al., 2020; Florentino y Ramírez, 2019).

Así también como en Europa, fue en España donde se encontró artículos relacionados los cuales brindan información acerca de los casos del COVID-19 en diferentes situaciones y escenarios. En estos trabajos de investigación fue necesario estudiar la dinámica de las epidemias que ayudan a comprender su crecimiento. Se observó también la implementación de un nuevo estado que son expuestos $E(t)$ donde se detalla el número de individuos que han sido afectados, pero no pueden infectar, lo cual lleva al modelo SEIR; este modelo solo se va a mencionar ya que nos centraremos en el modelo SIR (Barroso, 2020; Guirao, 2020; Aleja et al., 2020).

En Argentina, Speranza, Masci y otros autores, proponen una metodología de ajuste a las observaciones disponibles donde se usará el modelo SIR, que permita posteriormente proyectar un escenario de continuidad de la crisis sanitaria, en otro artículo se observa la aplicación del modelo matemático SIR donde se asume la inmunidad del recuperado pero que conforme pasa el tiempo se vuelve persona susceptible con lo cual este estudio no es certero; con ello, necesitaremos de otro modelo determinístico que se vería en otro tipo de investigación (Speranza y Masci, 2020; Bianco et al., 2020).

En las investigaciones de República Dominicana se observan resultados de simulaciones a través del modelo SIR de dos epidemias para comparar los resultados de la simulación con datos obtenidos experimentalmente; hoy en día los temas relacionados 
con la salud pública tienen un gran impacto y repercusión en la sociedad moderna, sobre todo si se trata de la propagación de una enfermedad infecciosa entre una extensa población (Florentino y Ramírez, 2019).

Por otra parte, Colombia se ha visto afectada por otras epidemias antes de la llegada del COVID-19 y gracias al uso del modelo matemático SIR o el uso de ecuaciones matemáticas se pudo comprender el comportamiento de dichas enfermedades debido a una extensa recopilación de información.

En donde se puede encontrar el desarrollo matemático del modelo SIR y su aplicación para predecir el curso de la pandemia por el COVID-19 es en la ciudad de Santa Marta en Colombia, con el fin de comprender todas las medidas de contención adoptadas por los Estados del mundo en la lucha contra la pandemia (Wilches y Castillo, 2020).

Así, el objetivo de esta investigación fue describir los diferentes enfoques y estrategias del modelamiento de evolución de enfermedades epidémicas basado en modelo epidemiológico SIR en los últimos años.

A partir de la revisión de la literatura, los modelos intentan tener una simulación lo más próxima y realista, para ello intervienen muchos factores que de acuerdo con el uso en el modelo pueden acercarse más o alejarse de la realidad. Por lo que se han venido haciendo estudios de varias enfermedades y viendo qué modelo se apega más a su comportamiento real, ya que las enfermedades tienen características distintas unas de otras. Con la llegada del SARS-CoV-2, el Perú ha sido afectado y duramente golpeado debido a la escasez de información y el desconocimiento del comportamiento de la enfermedad, lo que trajjo consigo lamentables pérdidas humanas, así como impactos en sectores sociales como económicos.

El adecuado estudio del modelo SIR podrá permitir tener un mayor conocimiento de la evolución del SARS-CoV-2 y así se pueda tener un soporte en la toma de decisiones por parte de las autoridades.

\section{MATERIALES Y MÉTODOS}

\subsection{Tipo de estudio}

Se realizó una revisión sistemática de la literatura científica con base en la adaptación de la metodología Prisma (Preferred Reporting Items for Systematic Reviews and MetaAnalyses) (Urrútia y Bonfill, 2010). La pregunta de investigación que se estableció para llevar a cabo el proceso metodológico fue la siguiente: ¿Cómo se describe el comportamiento de enfermedades epidémicas basado en modelo epidemiológico SIR en los últimos años? 


\subsection{Fundamentación de la metodología}

La revisión de la literatura es una fase imprescindible en trabajos de investigación, puesto que nos ayuda a situar la investigación y a sustentar teórica y conceptualmente a partir de lo escrito por investigadores e investigadoras sobre la temática. Se trata de identificar las aportaciones más importantes (pasadas y actuales) sobre el tema de estudio, así como definir los principales conceptos y teorías que sirvan para fundamentar y comprender el problema y valorar cómo se introduce en un marco más general de investigación (Arnau-Sabatés y Sala, 2020).

Existen investigaciones en las que la revisión de la literatura científica constituye en sí un método de investigación en que los datos son las aportaciones o los resultados de múltiples estudios que coinciden en una misma pregunta o tema de investigación, a pesar de que se hayan desarrollado con muestras distintas, en tiempos distintos, por diferentes equipos de investigación, etc. Dentro de este grupo se encuentran las revisiones sistemáticas, las scoping reviews y los estudios de metaanálisis, entre otras (Grant y Booth, 2009).

\subsection{Proceso de recolección de información}

Para garantizar la sensibilidad del proceso de búsqueda se definieron como descriptores los siguientes términos a partir de la pregunta de investigación: "modelo SIR", "modelos", "coronavirus", “modelos epidemiológicos”, "modelización”, “COVID-19". Por especificidad de la búsqueda de literatura científica, se diseñó un protocolo con la combinación de los términos establecidos y los operadores booleanos: [("modelo SIR" OR "modelos epidemiológicos") AND ("modelización" OR "modelos") AND ("enfermedades endemicas" OR "coronavirus")]. Igualmente, se definió como base de datos a EBSCOhost; como base de datos multidisciplinaria se eligieron Dialnet, Microsoft Academic y, como buscador genérico, se empleó Google Scholar.

Los artículos que fueron seleccionados se importaron al software de gestión de referencias Mendeley, para su gestión en la eliminación de citas duplicadas.

\subsection{Criterios de inclusión y de exclusión}

Como criterio de inclusión se consideraron artículos originales publicados en bases de datos científicas indexadas, en idioma inglés, portugués y castellano, entre los años 2011 y 2021, que describen el tema de un enfoque y estrategias del modelamiento de evolución de enfermedades endémicas basado en modelo epidemiológico SIR en los últimos años.

Por otra parte, como criterio de exclusión se definió que el abordaje de la revisión sistemática hubiera tomado como referencia un concepto que no está bien definido ni referenciado y no se brinde información verídica sobre las cantidades respectivas de cada variable requerida. 
El proceso de búsqueda y de extracción de información fue aplicado por tres revisores de forma independiente, cuyas diferencias fueron analizadas y resueltas por mutuo acuerdo.

\section{RESULTADOS}

La búsqueda de artículos en las bases de datos y motores de búsqueda arrojó un total de 53 artículos originales en el periodo de 2011 a 2021, distribuidos en repositorios bibliográficos como EBSCOhost, Dialnet y Google Scholar. A partir de este número total se eliminaron los duplicados con ayuda del gestor de referencias Mendeley. Posteriormente, se aplicaron criterios de inclusión y de exclusión hasta la obtención de un número final de 32 artículos para la presentación de resultados.

La ubicación geográfica de los países a los que pertenecen las universidades, instituciones educativas superiores y revistas de investigación identificadas en el estudio, evidencia que el tema de la modelización del comportamiento de enfermedades epidémicas es de interés global, y que se ha acentuado aún más con la llegada del COVID-19 en el mundo, como se puede apreciar en la figura 1.
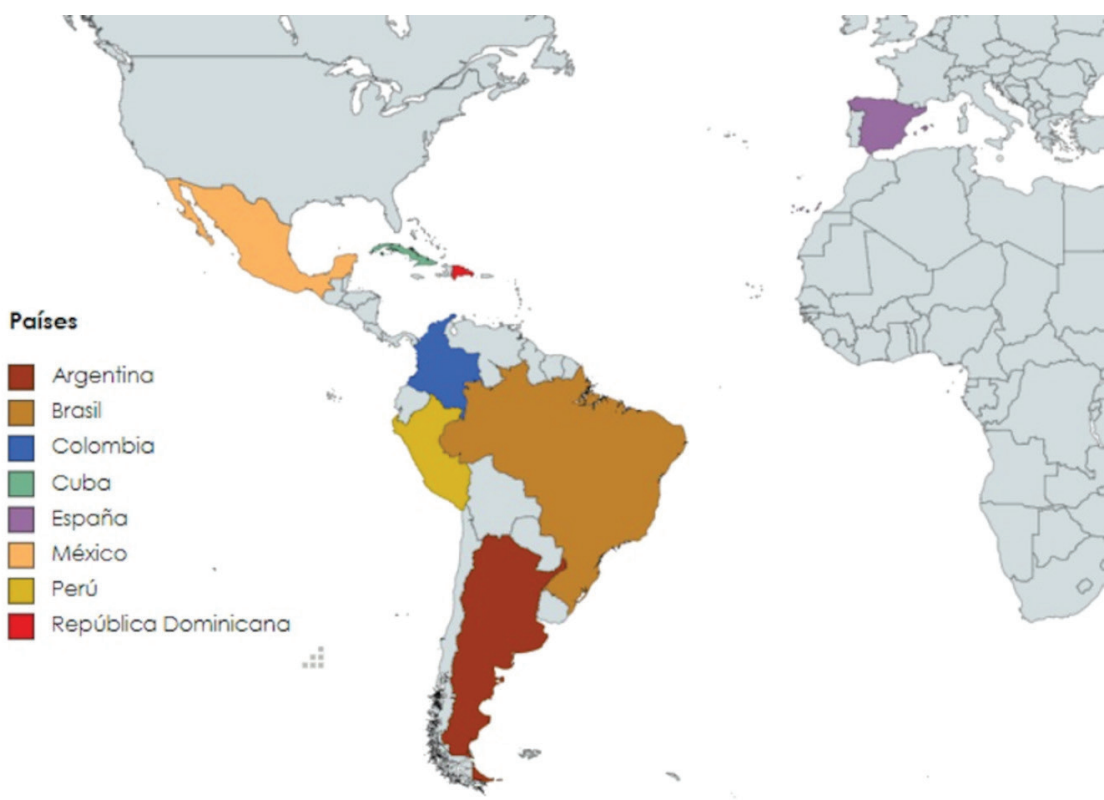

Figura 1. Ubicación geográfica de los países de la revisión de la literatura científica Elaboración propia 
Se encontró que los modelos de simulación epidemiológica han sido usados desde tiempos antiguos, como los aplicados en la epidemia en la isla de Bombay durante el periodo del 17 de diciembre de 1905 al 21 de Julio de 1906, mencionado por Pesco en el año 2017. Como se muestra en la figura 2, se tiene como datos los casos confirmados de peste que se presentaron (puntos negros) y la incidencia calculada por el modelo SIR (puntos blancos unidos a la curva). El gráfico muestra en el eje de las abscisas el tiempo en semanas, mientras que en el eje de las ordenadas el número de casos confirmados por semanas (Pesco, 2017).

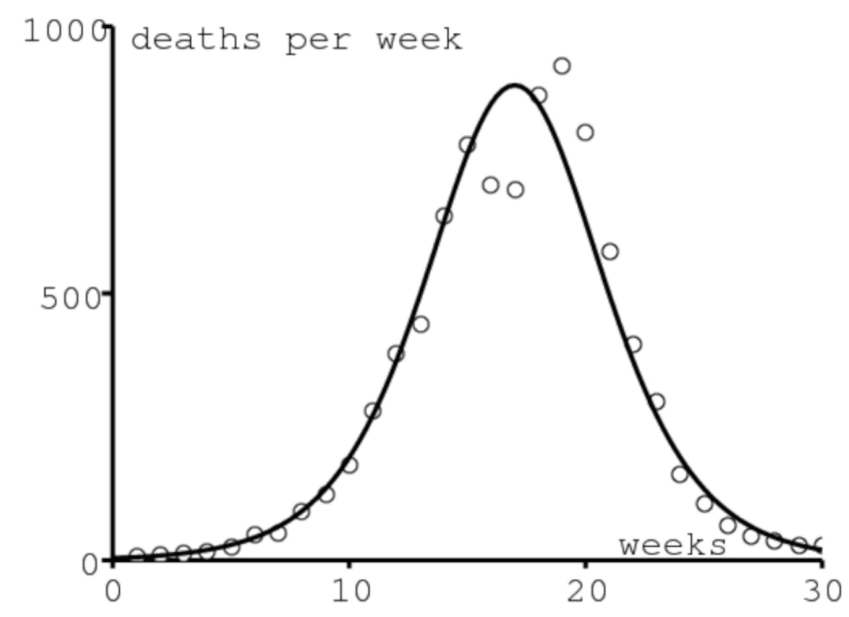

Figura 2. Casos de peste en la isla de Bombay en el año 1906

Fuente: Adaptado del trabajo de Pesco (2017)

Así, como también hoy en día con la aparición del COVID-19 se han venido realizando estudios de su comportamiento en diversos países del mundo, tales como lo indican Sanchez Peralta en la ciudad de México (figura 3), Manrique-Abril y otros en Colombia (figura 4), y Bezerra Sousa y otros en Brasil (figura 5) en sus respectivos artículos realizados en al año 2020 (Bezerra et al., 2020; Manrique-Abril et al., 2020; Sánchez, 2020). 


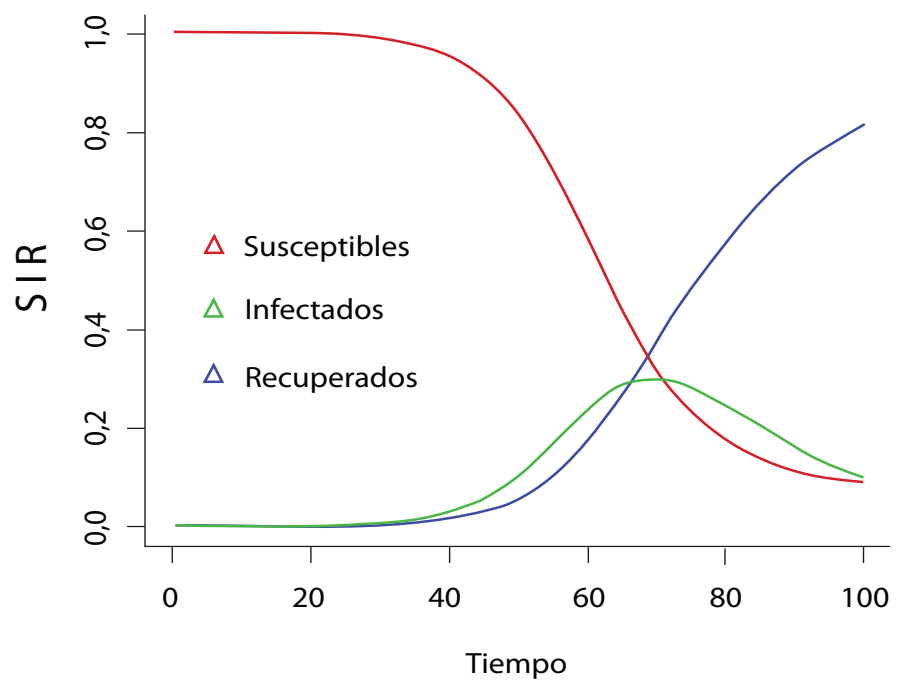

Figura 3. Modelo SIR del COVID-19 en la alcaldía de Iztapalapa

Fuente: Sánchez (2020)

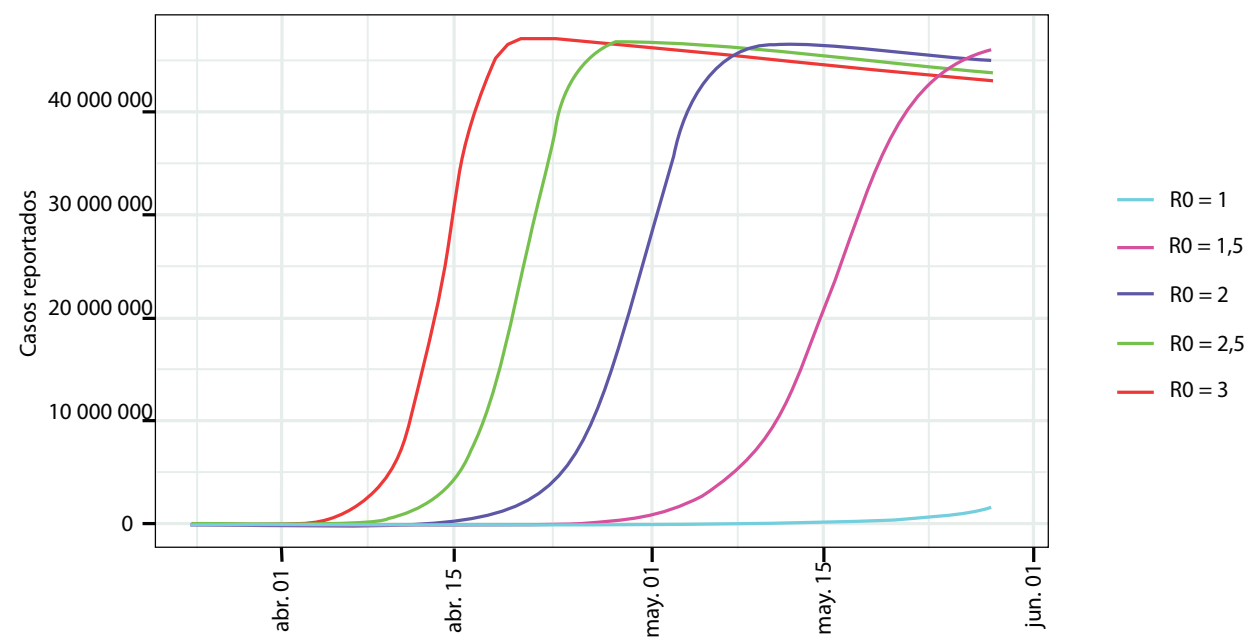

Figura 4 Modelo SIR de la evolución del COVID-19 en Colombia

Fuente: Manrique-Abril et al. (2020) 


\section{Río de Janeiro}

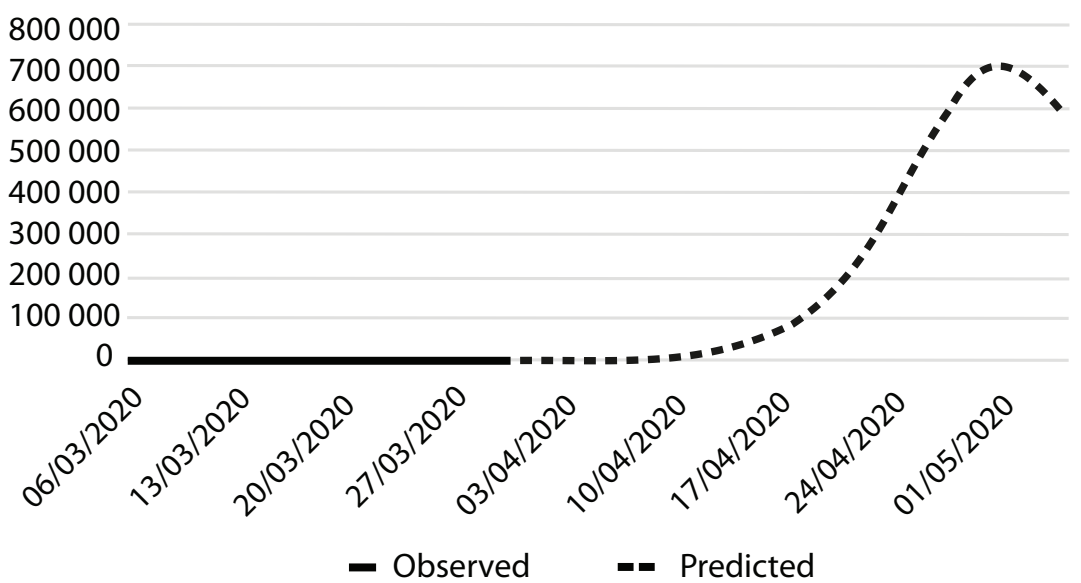

Figura 5. Modelo SIR aplicado en la ciudad de Río de Janeiro

Fuente: Bezerra et al. (2020)

Los gráficos del modelo SIR que se presentan fueron aplicadas en el periodo en el que empezó a emerger la enfermedad en los respectivos países.

En el Perú también se realizaron investigaciones acerca del uso del modelo SIR; autores como Gonzales-Castillo, Espinola-Sánchez, Vergara Moreno, Pino Romero, luego de haber realizado el modelo SIR y haber descrito el comportamiento del COVID-19, señalan que el modelo SIR sirve de apoyo en la toma de decisiones y que en el Perú se debe seguir tomando medidas de prevención para contener la pandemia, además de que, sin ellas, la enfermedad se hubiese propagado mucho más rápido (Gonzales-Castillo et al., 2020; Pino et al., 2020; Vergara et al., 2020; Espinola-Sánchez et al., 2020).

Se encontró en tesis y artículos un indicador importante en la modelización de enfermedades epidémicas; para los autores Osval A. Montesinos López y Carlos M. Hernández Suárez en el año 2007, citados en Pesco (2017), García (2014), Huamaní et al. (2020), el número reproductivo básico representa la capacidad reproductiva de la enfermedad. Además, está asociada la infectividad de la enfermedad y las características del contacto infectivo. Este parámetro es de mucho interés debido a que es un valor umbral que define el crecimiento o decrecimiento en la incidencia de la epidemia (García, 2014; Pesco, 2017; Huamaní et al., 2020).

En la revisión que se ha hecho de esta información se tiene que la estimación de la propagación de la pandemia por el COVID-19 en la ciudad de Santa Marta en Colombia se hace a partir de dos modelos SIR con parámetros y condiciones iniciales distintas (Wilches y Castillo, 2020). Como se muestra en la figura 6, todos los casos reportados 
por el COVID-19 con respecto a la fecha en donde fueron reportados en este gráfico se representa para distintos valores de $R_{e}$.

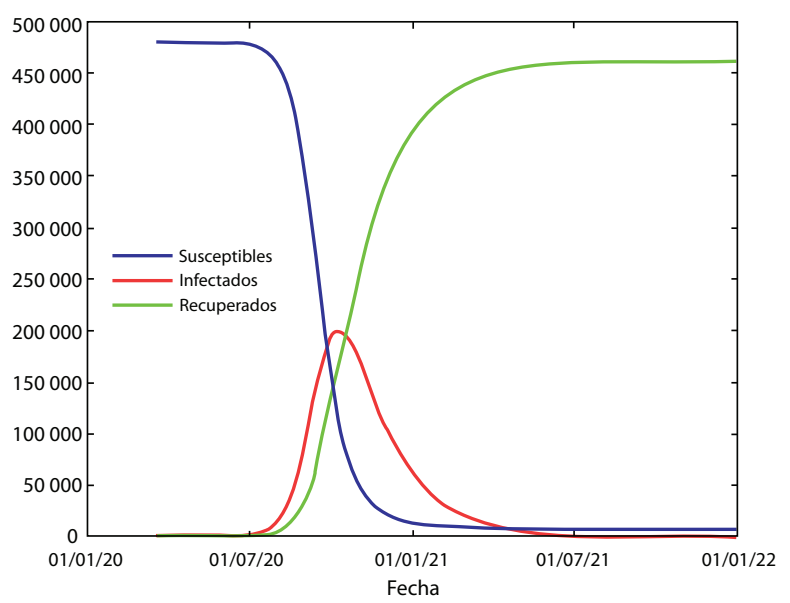

Figura 6. Casos reportados y estimados para la pandemia por el COVID-19 en Santa Marta, Colombia, para distintos valores de Re

Fuente: Wilches y Castillo (2020)

De la figura 6 se infiere que, cuanto mayor valor posee el $R_{e}$ (la tasa de reproducción básica), más rápidamente se incrementa la cantidad de casos estimados en el tiempo (Wilches y Castillo, 2020).

Se encontró un reporte detallado del COVID-19 que se desarrolló en una ciudad de Colombia en el que los afectados fueron clasificados por susceptibles, infectados y recuperados como se muestra en la figura 7 , donde se observa que la cantidad de susceptibles se proyecta a bajar considerablemente; la cantidad de infectados llegó a su pico más alto aproximadamente en octubre o noviembre pero igualmente se proyecta a bajar; en cambio, los recuperados al inicio eran pocos pero con los cuidados que se ha ido dando ha subido considerablemente. 


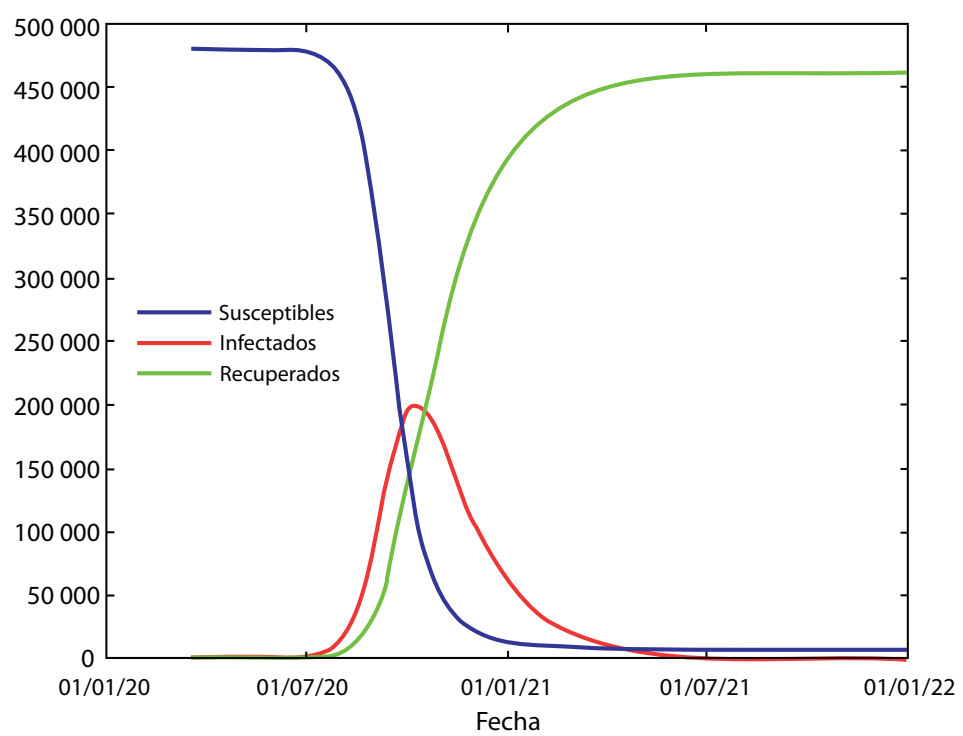

Figura 7. Curva de la cantidad de susceptibles, infectados y recuperados estimados para la pandemia por el COVID-19 en Santa Marta, Colombia

Fuente: Wilches y Castillo (2020)

La cantidad máxima de infectados simultáneamente corresponde a casi el $42 \%$ de la población total y se registraría el 9 de octubre de 2020 . Por otro lado, cerca del 98,5\% de la población llegaría a infectarse y poco más del $96 \%$ se recuperarán. En este modelo se supuso que los recuperados no volverán a infectarse, por lo que la curva de susceptibles es siempre decreciente en el tiempo (Wilches y Castillo, 2020).

Autores como Barroso en el año 2020 hizo una aplicación del modelo SIR al caso del COVID-19 en España. Se han utilizado los datos disponibles sobre el número de infectados por el COVID-19 en España, procedentes del Centro Europeo para el Control y Prevención de Enfermedades. La serie de datos está disponible desde el 4 de febrero del 2020 hasta el 5 de abril del 2020, como se muestra en la figura 8 , en donde se presentan las fechas en que han sido infectadas respecto a los casos acumulados y se observan los puntos azules que indican los valores reales y la línea roja que muestra la predicción modelo SIR (Barroso, 2019). 


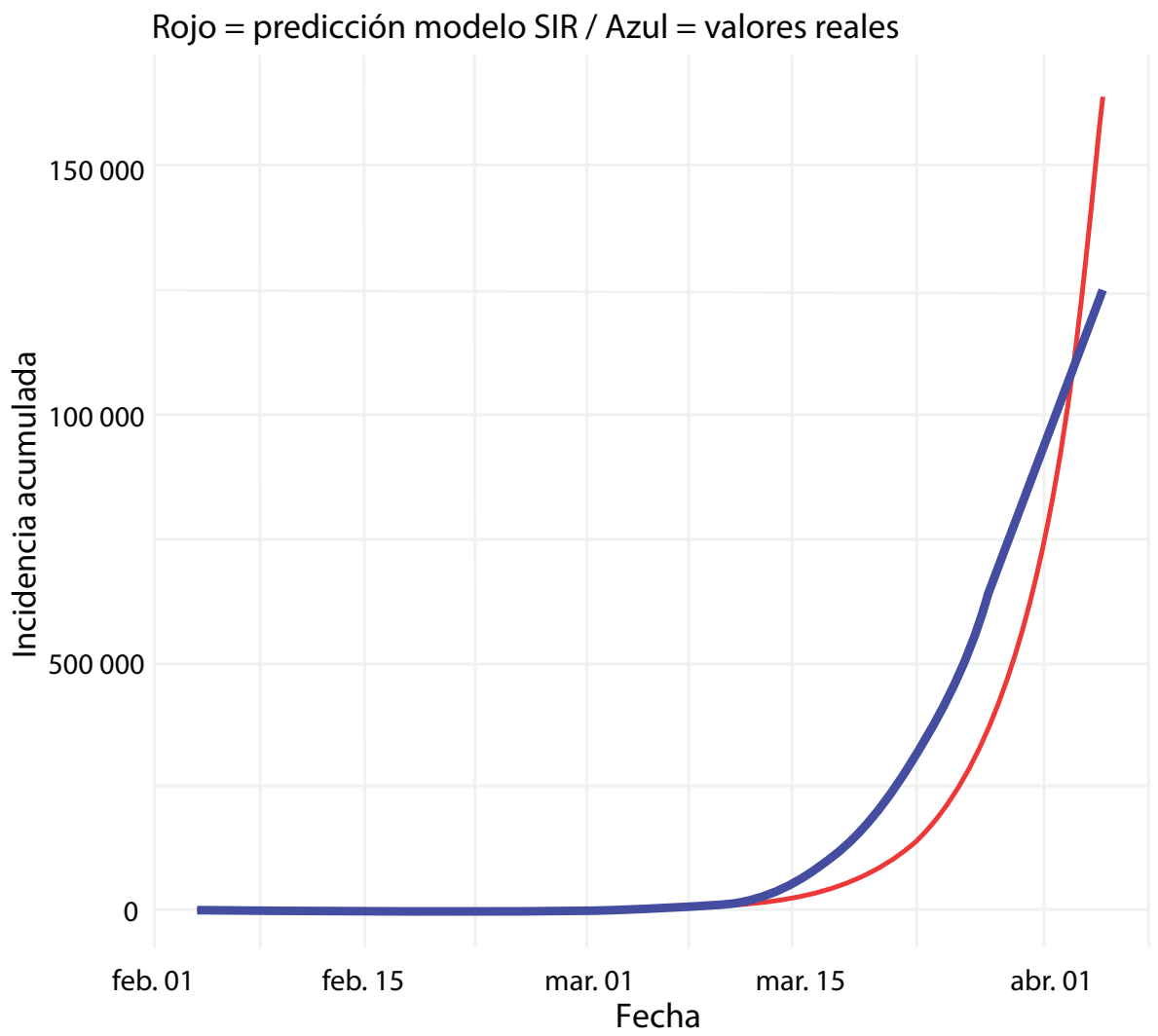

Figura 8. Estimación del número acumulado de personas infectadas en España

Fuente: Barroso (2019)

En el caso de Argentina se encontró que el modelo SIR permite la proyección a partir de la calibración inicial con los parámetros que explican la evolución de la enfermedad, luego se realizó una proyección respecto de los treinta días posteriores al último dato observado, de esta manera se obtuvo el resultado que se presenta en la figura 9 (Speranza y Masci, 2020). 


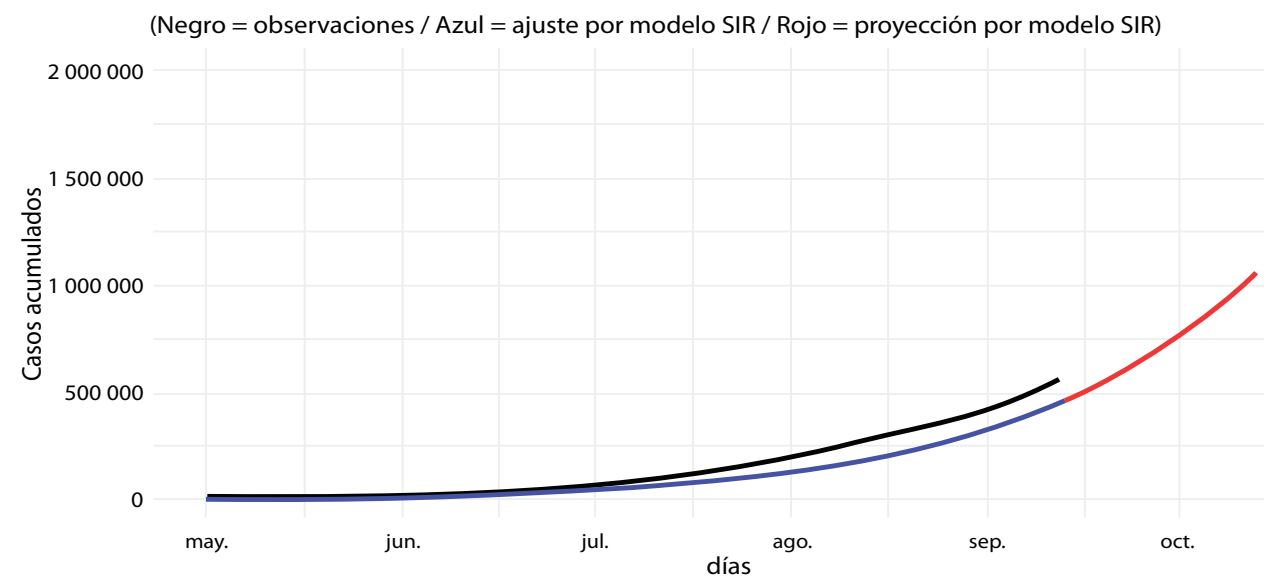

Figura 9. Ajuste y proyección mediante el modelo SIR de casos totales confirmados acumulados del COVID-19 en Argentina

Fuente: Tomado de Speranza y Masci (2020)

El COVID-19, única pandemia de coronavirus registrada en la historia de la humanidad, ha dejado un saldo funesto de contagiados y muertos a escala global a lo largo de los últimos años desde su aparición en el mundo.

Por último, a través de las ecuaciones del modelo SIR y su corroboración en el ejemplo citado se puede explicar por qué la cuarentena, el distanciamiento físico y la búsqueda de tratamientos efectivos y vacunas han recibido toda la atención mediática y han focalizado esfuerzos económicos ingentes por parte de todos los países del mundo.

\section{DISCUSIÓN}

La revisión de la literatura científica realizada nos ubica en un contexto en el cual se viene realizando diversidad de investigaciones e implementación del modelo SIR para modelar la pandemia provocada por el COVID-19. Como se ha podido revisar de las investigaciones que han realizado en diferentes países, el modelo se ha aplicado en variedad de artículos para modelar otras enfermedades epidémicas de origen viral y que han permitido que el modelo esté presente en los estudios en el campo de la epidemiología (Pesco, 2017; Manrique-Abril et al., 2020; Bezerra et al., 2020; Sánchez, 2020).

En la revisión realizada, varios autores mencionaron el uso del indicador básico de reproducción obtenido del modelo SIR y que muestra la evolución teniendo una variación entre 0 y 1 cuando la enfermedad empieza a disminuir; este indicador en artículos de investigación mostró cómo las medidas adoptadas por los gobiernos para la contención de enfermedades han ayudado a suprimir el contagio y evitar la expansión de la 
enfermedad en la población (Pliego, 2011; Vergara et al., 2020; Yanqui, 2018). Además, en los artículos revisados se proporcionan algunas recomendaciones sobre las medidas adoptadas por el gobierno para que se pueda seguir reteniendo la propagación de la enfermedad, como lo es el lavado de manos, la distancia social, entre otras (Sánchez, 2020; Martínez et al., 2015).

Lo encontrado en las investigaciones explica cómo se propaga rápidamente el contagio del COVID-19 y poder predecir este comportamiento; los resultados mostraron que este modelo garantiza una menor cantidad de infectados totales y diarios, relacionados con una distribución de infectados más homogénea, con lo cual la probabilidad de colapso hospitalario es menor. Se puede aplicar el modelo agregando nuevas poblaciones epidemiológicas y factores en el modelo (Wilches y Castillo, 2020).

En el caso de España, en comparación con los otros países ya mencionados, el modelo predice un crecimiento de la enfermedad más acelerado que el realmente observado. Esta diferencia podría deberse a las distintas medidas de confinamiento y limitaciones de la actividad económica que se han ido produciendo en España desde el 11 de marzo del 2020, algo similar sucede en Argentina al utilizar el modelo que ayuda a proveer detalles y predicciones de la diseminación del virus en una comunidad, o como en este caso en un país, mientras que los datos en su estado natural no permiten proveer esta información (Speranza y Masci 2020; Barroso, 2020).

\section{CONCLUSIONES}

A partir de la revisión sistemática que se realizó en variedad de repositorios académicos usando la metodología Prisma, se concluye que el modelo SIR es un modelo aplicado por muchos investigadores para modelar el comportamiento de enfermedades epidémicas y que a raíz de la aparición de la pandemia ha incrementado el interés de su implementación. Se ha llegado a usar el modelo SIR en enfermedades como la gripe, influenza y coronavirus en diferentes países en el continente americano y Europa. Ello permite que se cuente con un modelo que apoya en la toma de decisiones a las autoridades para mitigar el impacto que genera las epidemias en la sociedad.

Se concluye, sobre la base de la revisión sistemática, que el modelo SIR permite estimar el número básico de reproducción, el cual permite saber la cantidad de infectados que puede ocasionar un solo infectado en promedio. Este valor es usado como indicador en la evolución de la pandemia en variedad de artículos y tesis. El valor de $\mathrm{R}_{\mathrm{e}}$ al ser menor a 1, determina que la epidemia descenderá hasta inclusive llegar a extinguirse, de lo contrario, al ser su valor mayor a 1, significa que la epidemia seguirá en ascenso.

En la revisión de artículos y tesis, en el caso de Colombia se concluye que gracias al modelo SIR se pudo implementar los mecanismos de lucha contra la pandemia, 
tales medidas como el distanciamiento social, la búsqueda de tratamientos efectivos y vacunas. Gracias a esto se ha logrado reducir el nivel de la tasa de mortalidad y obtener menos contagios a nivel nacional.

En el caso de España se puede decir que por el modelo SIR aplicado, la predicción realizada por el modelo presenta un crecimiento más acelerado que el real, esto se debe a las diferentes medidas que toma el gobierno con respecto al COVID-19 como la cuarentena y las restricciones de trabajo de la población las cuales se han visto afectadas desde que empezó esta pandemia, también afecta el cuidado que tienen las personas con respecto a la protección e higiene.

Se concluye en el caso de Argentina que el modelo desarrollado que se ha aplicado permite proveer predicciones de cómo avanza el virus en la comunidad, mientras se compara con los datos reales y así determinar qué tan acertada puede ser la aplicación de este modelo en enfermedades epidémicas; al igual que en España estos datos sirven para proyectar estimaciones sobre los números de casos futuros para así prevenir y tomar medidas la cual ayuden a salir de estas enfermedades epidémicas.

En general los modelos epidemiológicos planteados de diferentes países son herramientas útiles y beneficiosas que busca representar el comportamiento de un virus o como en este caso una pandemia, dado que nos ayuda a construir una predicción en base a su evolución, para así tomar medidas contra dicha enfermedad.

Sobre la metodología empleada para realizar esta investigación es importante aclarar que las revisiones sistemáticas emergieron en el ámbito de la epidemiología, pero que con el avance de la computación su utilidad ha llevado a que se apliquen en otras áreas.

\section{REFERENCIAS}

Abelló Ugalde, I. A., Guinovart Díaz, R.,y Morales Lezca, W. (2020). El modelo SIR básico y políticas antiepidémicas de salud pública para el COVID-19 en Cuba. Revista Cubana de Salud Pública, 46(Supl. especial). https://www.scielosp.org/article/ rcsp/2020.v46suppl1/e2597/

Aleja D, Criado R., y Romance M. (2020). Modelo SEIR para el Coronavirus COVID-19. https://www.dcncsciences.com/wp-content/uploads/2020/04/Informe-1.pdf

Arnau-Sabatés, L., y Sala Roca, J. (2020). La revisión de la literatura científica: Pautas, procedimientos y criterios de calidad. Departamento de Teorías de la Educación y Pedagogía Social, Universidad Autónoma de Barcelona. https://docplayer. es/192625327-La-revision-de-la-literatura-cientifica-pautas-procedimientosy-criterios-de-calidad.html 
Barroso, V. M. (2020). Una breve introducción al modelo SIR aplicado al caso del COVID-19. ICEI Papers COVID-19, 12. https://www.ucm.es/icei/file/iceipapercovid12.

Bezerra Sousa, G., Santos Garces, T., Feitosa Cestari V., Magalhães Moreira, T., Sampaio Florêncio, R., y Duarte Pereira, M. L. (2020). Estimación y predicción de casos de COVID-19 en metrópolis brasileñas. Revista Latino-Americana de Enfermagem, 28. https://doi.org/10.1590/1518-8345.4501.3345

Bianco, M. J., Cruz, P. D., Fraquelli, A. D., y Gache, A. L. (2020). Modelo epidemiológico SIR: Una aplicación de las ecuaciones diferenciales al SARS-CoV-2 (COVID-19). Revista de Investigación en Modelos Matemáticos aplicados a la Gestión y la Economía, 7(1), 16-38. http://www.economicas.uba.ar/wp-content/uploads/2016/04/Gache-Andrea-.pdf

Cabo Bizet, N., y Cabo Montes de Oca, A. (2020). Modelos SIR modificados para la evolución del COVID-19. Revista De Información Científica Para La Dirección En Salud (INFODIR): Ciencias Matemáticas 34(1), 73-87. http://www.revinfodir.sld.cu/ index.php/infodir/article/view/1041

Catano-Lopez, A., y Rojas-Diaz, D. (2020). Modelos discretos de transmisión del COVID-19 y publicaciones preliminares en la ciencia: una búsqueda sistematizada. Scielo Preprint, 1-15. https://doi.org/10.1590/SciELOPreprints.1076

Espinola-Sánchez, M., Racchumí-Vela, A., Sanca-Valeriano, S., Espinola-Sánchez, S., Arango-Ochante, P., Saldaña-Díaz, C., Segundo-Paredes, J., y Mejico-Caja, M. (2020). Pandemia del COVID-19 y efecto de medidas de contención en población Peruana: Un modelamiento matemático SIR. Revista Del Cuerpo Médico Hospital Nacional Almanzor Aguinaga Asenjo, 13(2), 110 - 115. https://doi.org/10.35434/ rcmhnaaa.2020.132.656

Florentino Lorenzo, A., y Ramírez Contrera, F. (2019). Simulación de dos enfermedades epidemiológicas de República Dominicana a través del modelo SIR. ROCA: Revista científico-educacional de la provincia Granma, 15(4), 1-10. https://revistas. udg.co.cu/index.php/roca/article/view/1013

Galindo Uribarri, S., Rodríguez Meza, M., y Cervantes Cota, J. (2013). Las matemáticas de las epidemias: caso México 2009 y otros. CIENCIA Ergo-Sum, 20(3), 238-246. https://www.redalyc.org/pdf/104/10428759009.pdf

Garcia Piñera A. (2014). Modelos de ecuaciones diferenciales para la propagación de enfermedades infecciosas [Trabajo de fin de grado, Unidad de Cantabria]. Repositorio Abierto de la Universidad de Cantabria. http://hdl.handle. net/10902/7125

Gonzales-Castillo, J. R., Varona-Castillo, L., Domínguez-Morante, M. G., y Ocaña-Gutierrez, V. R. (2020). Pandemia del COVID-19 y las Políticas de Salud Pública en el Perú: 
marzo-mayo 2020. Revista de Salud Pública, 22(2), 1-9. https://doi.org/10.15446/ rsap.v22n2.87373

Grant, M. J., y Booth, A. (2009). A typology of reviews: An analysis of 14 review types and associated methodologies. Health Information y Libraries Journal, 26, 91-108. https://doi.org/10.1111/j.1471-1842.2009.00848.x

Grillo Ardila, E. K., Bravo Ocaña, L. E., Guerrero, R., y Santaella-Tenorio, J. (2020). Mathematical models and the coronavirus, COVID-19. Colombia Médica, 51(2). https://doi.org/10.25100/cm.v51i2.4277

Guirao Piñera, A. (2020). Entender una epidemia: El coronavirus en España, situación y escenarios. Revista Española de Física, 34(2), 3-10. http://www.revistadefisica. es/index.php/ref/article/view/2659

Gutiérrez, J. M., y Varona, J. L. (2020). Análisis de la posible evolución de la epidemia de coronavirus COVID-19 por medio de un modelo SEIR. Universidad de La Rioja: Departamento de Matemáticas y Computación, 1-14. https://www.unirioja.es/ apnoticias/servlet/Archivo?C_BINARIO=12051

Huamaní C, Timaná-Ruiz R, Pinedo J, Pérez J., y Vásquez L. (2020). Condiciones estimadas para controlar la pandemia del COVID-19 en escenarios de pre y poscuarentena en el Perú. Revista Peruana de Medicina Experimental y Salud Pública, 37(2), 195-202

Martínez, F., Gil, G., Guzmán, N., Castañeda, T., Buitrago, N., Bohórquez, y E., Mura, I. (2015). Modelado predictivo de la epidemia de Chikungunya. Revista Ontare, 3(2), 7-31 . https://doi.org/10.21158/23823399.v3.n2.2015.1439

Manrique-Abril, F. G., Agudelo-Calderon, C. A., González-Chordá, V. M., GutiérrezLesmes, O., Téllez-Piñerez, C. F., y Herrera-Amaya, G. (2020). Modelo SIR de la pandemia del COVID-19 en Colombia. Revista de Salud Pública, 22(2), 1-9. https:// doi.org/10.15446/rsap.v22n2.85977

Pesco P. (2017). Modelos estocásticos para epidemias recurrentes [Tesis de doctorado, Universidad Nacional de la Plata]. Repositorio Institucional de la UNLP. https:// doi.org/10.35537/10915/64320

Pino Romero, N., Soto-Becerra, P. y Quispe Mendizábal, R. A. (2020). Un Modelo Matemático SIR-D Segmentado para la Dinámica de Propagación del Coronavirus (COVID-19) en el Perú. Selecciones Matemáticas, 7(1), 162-171. https://doi.org/10.17268/sel. mat.2020.01.15

Pliego Pliego, E. C. (2011). Modelos Epidemiológicos de Enfermedades Virales Infecciosas [Tesis de licenciatura]. Benemérita Universidad Autónoma de Puebla. 
https://www.fcfm.buap.mx/assets/docs/docencia/tesis/matematicas/ EmileneCarmelitaPliegoPliego.pdf

Sánchez, A. (2020). Un estudio del COVID-19 en Iztapalapa usando el modelo SIR. INEGI. https://www.researchgate.net/publication/342132072_Un_estudio_del_Covid-

Sepúlveda, L. (2015). Manejo optimo y viable en modelos epidemiológicos de dengue [Tesis de doctorado]. Université Paris-Est; Universidad nacional de Colombia. https://pastel.archives-ouvertes.fr/tel-01376000/document

Speranza, M. E., y Masci, M. E. (2020). Metodología de ajustamiento y proyección S.I.R. a la cantidad de infectados en pandemia: El caso del COVID-19 en Argentina. 40 Jornadas Nacionales de Administración Financiera, 273- 282. https://economicas. unsa.edu.ar/afinan/informacion_general/sadaf/xl_jornadas/40-j-speranzamasci-metodologia-de-ajustamiento-y-proyeccion-s.i.r.pdf

WHO. (2020). Novel Coronavirus (2019-nCoV): Situation Report- 1. https://www.who.int/ docs/default-source/coronaviruse/situation-reports/20200121-sitrep-1-2019ncov.pdf?sfvrsn=20a99c10_4

WHO. (2020). Coronavirus Disease (COVID-19) Situation Reports 2020. https://www.who.int/ emergencies/diseases/novel-coronavirus-2019/situation-reports

Urrútia G., y Bonfill X. (2010). Declaración PRISMA: una propuesta para mejorar la publicación de revisiones sistemáticas y metaanálisis. Medicina Clínica, 135(11), 507-511. https://doi.org/10.1016/j.medcli.2010.01.015

Vergara Moreno, E., León Navarro, R., More Ayala, J., Arteaga Blas, D., Asmat Uceda, R., Peralta Castañeda, J., Quito Santos, C., Vargas Pichon, H., y Rubio, O. (2020). Modelo básico epidemiológico SIR para el COVID-19: caso las Regiones del Perú. Selecciones Matemáticas, 7(1), 151-161. https://doi.org/10.17268/sel. mat.2020.01.14

Wilches Visbal, J. H., y Castillo Pedraza, M.C. (2020). Aproximación matemática del modelo epidemiológico SIR para la comprensión de las medidas de contención contra el COVID-19. Revista Española de Salud Pública 94, 23. https://www.mscbs. gob.es/biblioPublic/publicaciones/recursos_propios/resp/revista_cdrom/ VOL94/C_ESPECIALES/RS94C_202009109.pdf

Yanqui Diaz, F. (2018). Simulación epidemiológica usando el modelo SEIR del brote de influenza A H1N1 en el distrito de Abancay [Tesis de maestría, Universidad Nacional del Altiplano]. Repositorio Institucional Digital de la Universidad Nacional del Altiplano. http://repositorio.unap.edu.pe/handle/UNAP/8448 